\title{
Non-inflammatory tumor microenvironment of diffuse intrinsic pontine glioma
}

\author{
Grant L. Lin', Surya Nagaraja', Mariella G. Filbin², Mario L. Suvà ${ }^{3,4}$, Hannes Vogel ${ }^{1,5,6}$ and Michelle Monje ${ }^{1,5,6^{*}}$ (D)
}

\begin{abstract}
Diffuse intrinsic pontine glioma (DIPG) is a universally fatal malignancy of the childhood central nervous system, with a median overall survival of 9-11 months. We have previously shown that primary DIPG tissue contains numerous tumor-associated macrophages, and substantial work has demonstrated a significant pathological role for adult glioma-associated macrophages. However, work over the past decade has highlighted many molecular and genomic differences between pediatric and adult high-grade gliomas. Thus, we directly compared inflammatory characteristics of DIPG and adult glioblastoma (GBM). We found that the leukocyte (CD45+) compartment in primary DIPG tissue samples is predominantly composed of CD11b + macrophages, with very few CD3+ T-lymphocytes. In contrast, T-lymphocytes are more abundant in adult GBM tissue samples. RNA sequencing of macrophages isolated from primary tumor samples revealed that DIPG- and adult GBM-associated macrophages both express gene programs related to ECM remodeling and angiogenesis, but DIPG-associated macrophages express substantially fewer inflammatory factors than their adult GBM counterparts. Examining the secretome of glioma cells, we found that patient-derived DIPG cell cultures secrete markedly fewer cytokines and chemokines than patient-derived adult GBM cultures. Concordantly, bulk and single-cell RNA sequencing data indicates low to absent expression of chemokines and cytokines in DIPG. Together, these observations suggest that the inflammatory milieu of the DIPG tumor microenvironment is fundamentally different than adult GBM. The low intrinsic inflammatory signature of DIPG cells may contribute to the lack of lymphocytes and non-inflammatory phenotype of DIPG-associated microglia/macrophages. Understanding the glioma subtype-specific inflammatory milieu may inform the design and application of immunotherapy-based treatments.
\end{abstract}

Keywords: Diffuse intrinsic pontine glioma, Tumor-associated macrophage, Tumor-infiltrating lymphocyte, Glioma, Glioblastoma, Immune microenvironment

\section{Introduction}

Diffuse intrinsic pontine glioma (DIPG) is a devastating tumor that arises in the ventral pons, chiefly during childhood, and is universally fatal. Currently, the standard of care is radiotherapy, which offers temporary stabilization or reduction of symptoms and extends median survival by approximately 3 months [21]. Overall median survival remains 9-11 months with less than $10 \%$ of children surviving beyond 2 years $[7,20]$. Over the past decade, comprehensive efforts to characterize

\footnotetext{
* Correspondence: mmonje@stanford.edu

${ }^{1}$ Department of Neurology, Stanford University, Stanford, CA 94305, USA ${ }^{5}$ Department of Pathology, Stanford University, Stanford, CA 94305, USA Full list of author information is available at the end of the article
}

the genomic and epigenomic features of DIPG and other pediatric tumors have revolutionized our understanding of this disease [29]. Pediatric high-grade gliomas are genomically distinct tumors when compared to adult glioblastoma (GBM) [47]. A highly recurrent mutation in genes encoding histone-3 (H3K27M) is found in $\sim 80 \%$ of all DIPG and also the majority of thalamic and spinal cord gliomas of childhood [2, 9, 11, 22, 44, 45, 48, 50, 53, 54]; these H3K27M mutant gliomas were recently reclassified as "diffuse midline gliomas, H3K27M mutant subtype" [23]. Broadly, analysis of immune infiltrates of pediatric central nervous system tumors suggests the immune compartments are less predictive of tumor grade or prognosis, compared to adult gliomas [37]. For example, pediatric

(c) The Author(s). 2018 Open Access This article is distributed under the terms of the Creative Commons Attribution 4.0 International License (http://creativecommons.org/licenses/by/4.0/), which permits unrestricted use, distribution, and 
GBM samples exhibit a muted immune functional phenotype compared to pediatric pilocytic astrocytomas and ependymomas [15], but the immune state of DIPG has not been similarly analyzed to date. DIPG exhibits a unique mutational profile and transcriptional state [27, 29], raising the question of a similarly unique immune microenvironment. As interest in the clinical application of immunotherapeutic approaches for DIPG grows, it is critically important to understand how potential differences in the DIPG immune microenvironment could influence treatment efficacy. For example, the presence or absence of lymphocytes would inform the consideration of strategies such as checkpoint inhibition.

Glioma-associated microglia/macrophages (GAMs) have received interest as a potential therapeutic target. In patients with adult GBM, expression of specific immune-associated gene sets are correlated with overall survival [52]. Moreover, adult GBM-associated macrophages have been implicated in supporting tumor cell proliferation, invasion, and survival as well as tumor angiogenesis [13, 17]. Targeting GAMs with CSF1R antagonism has demonstrated efficacy in a number of adult GBM preclinical models by affecting GAM activation state [39], an effect mediated by the tumor microenvironment [41]. CSF1R antagonism also potentiates the effect of radiotherapy in adult GBM preclinical models [46]. While evaluation of the clinical potential of CSF1R- and other GAM-targeting therapies is ongoing $[3,5]$, it is clear that GAMs are a critical component of the glioma microenvironment. Moreover, GAM activation state has been identified as an important factor in effective immunotherapy treatment [43], so understanding their phenotype across different tumors may help guide clinical translation of immune-targeting therapies.

In DIPG, little is known about the state of microglia and macrophages: due to the sensitive location and diffusely infiltrative nature of the tumor, resection is not possible, and the biopsy of DIPG has historically been uncommon [4, 23, $29,38]$. We have previously demonstrated that human DIPG samples demonstrate substantial immunoreactivity for the macrophage/microglial markers CD68 and CD163 [6], illustrating that GAMs are a large fractional component of the DIPG microenvironment. Here, we isolate GAMs from primary DIPG and adult GBM, as well as microglia from normal pediatric cortex samples for whole transcriptome analysis. We demonstrate that while DIPG-associated macrophages exhibit some gene expression programs similar to adult GBM-associated macrophages, they express substantially fewer inflammatory cytokines and chemokines compared to adult GAMs. Proteomic analyses reveal that patient-derived DIPG cultures produce markedly fewer cytokines and chemokines when compared to patient-derived adult GBM cultures, a finding corroborated by primary DIPG tissue bulk and single-cell RNA sequencing data. We also observed minimal lymphocytic infiltration in both primary DIPG autopsy and diagnostic biopsy samples. Together, these data suggest that DIPG and DIPG-associated macrophages are less inflammatory than adult GBM and adult GAMs.

\section{Materials and methods}

Acquisition and processing of human tissue samples

All human tissue studies were performed with informed consent and in accordance with Institutional Review Board (IRB)-approved protocols. Autopsy and biopsy tissue samples were processed as previously described [26], with some modifications. Briefly, tissue was minced finely, enzymatically dissociated in a collagenase/dispase + DNAse solution, triturated, and filtered through a $100 \mu \mathrm{m}$ filter to obtain a single cell solution. Debris was removed by centrifugation using a $0.9 \mathrm{M}$ sucrose gradient, and red blood cells were removed using ACK lysis. Live cells were quantified via trypan blue exclusion, and resuspended for fluorescence-activated analysis and sorting.

\section{Fluorescence-activated cell sorting and analysis}

Isolated single cells were resuspended in flow cytometry buffer (2\% BSA, 10 $\mu$ M HEPES in HBSS without calcium/ magnesium) at approximately $10^{7}$ cells $/ \mathrm{mL}$ and processed at $4{ }^{\circ} \mathrm{C}$. Cells were blocked with mouse IgG isotype control (ThermoFisher) and rat IgG isotype control (R\&D Systems) as appropriate. Cells were then incubated with the appropriate conjugated primary antibodies (FITC-anti-CD45, BD Pharmigen 555482; PE-Cy7-anti-CD11b, BD Pharmigen 557743; PE-anti-CD3, Biolegend 300308; APC-Cy7-anti-CD31, Biolegend 303120) for 1 h. Next, cells were stained using the LIVE/DEAD Fixable Dead Cell Stain Kit (Invitrogen) according to the manufacturer's instructions. Immediately before analysis and sorting, DAPI was added to the flow cytometry buffer. Cells were sorted and analyzed on a BD FACSAria II or FACSAria Fusion. Cells were identified and gated for size, singularity, cell viability, and all surface-marker expression was gated with appropriate full-minus-one controls. Cells were sorted into cold catching medium and a fraction was removed for cell purity analysis. Isolated cells were lysed in TRIzol (Invitrogen), and stored at $-80^{\circ} \mathrm{C}$ until further processing.

\section{RNA sequencing}

Total RNA was extracted by chloroform extraction into the RNA Clean \& Concentrator Kit with DNAse (Zymo Research), and examined for quality using an Agilent Bioanalyzer 2100 platform. First and second-strand cDNA synthesis and SPIA amplification was performed with the Ovation RNAseq system V2 (Nugen) following the manufacturer's instructions, and the resulting cDNA was fragmented with a sonicator (Covaris) using the following parameters: duty cycle $10 \%$, peak power 175.0 , cycles/burst 100, time $5 \mathrm{~min}$. 
Libraries were prepared for sequencing as previously described [35]. DNA was end repaired using T4 polymerase, Klenow fragment, and T4 polynucleotide kinase. 3' A-tailing was performed using Exo-Klenow. NEBNext Illumina Multiplex Oligo Adaptors (NEB E7335S) were ligated for $1 \mathrm{~h}$ at room temperature. Unligated adapters were separated by gel electrophoresis (2.5\% agarose, $0.5 \mathrm{X} \mathrm{TBE}$ ) and ligated DNA was purified using a NucleoSpin Gel Clean-up Kit (Macherey-Nagel). Ligated DNA was PCR amplified using NEBNext Multiplex Primers and purified using AMPure XP beads (Beckman Coulter). Purified libraries were quantified using Agilent 2100 Bioanalyzer HS DNA and multiplexed in equimolar concentrations. Sequencing was performed using an Illumina NextSeq at $1 \times 75$ bp by Stanford Functional Genomics Facility. RNA sequencing data is available through GEO (GSE115397).

Gene expression of patient-derived DIPG cultures and primary bulk DIPG tissue was published previously [11, 30, 35]. Single-cell RNA sequencing of diagnostic DIPG biopsies was also published previously [7]. All additional analysis was performed in R; FPKM plots were plotted in Prism (GraphPad).

\section{Gene expression analysis}

Reads were mapped to hg19 annotation using Tophat2 [24]. Transcript coverage of RefSeq gene annotations were performed using featureCounts [25]. To visualize sample similarity between samples, we performed a regularized log-transformation using the $\mathrm{R}$ function rlog from DESeq2 [28], and plotted the PCA using ggplot2. Differential testing and $\log 2$ fold change calculation was performed using DESeq2, with $F D R=0.1$ and normalization calculated by number of assigned reads. Active transcripts were defined as genes with a mean FPKM of at least 5 across all samples. Volcano plot was made in R. Gene Ontology on differentially expressed gene lists was performed using the DAVID v6.8 web portal $[18,19]$. Pre-ranked gene set enrichment analysis was performed with the publically available software platform $[33,49]$.

\section{Human tissue culture}

Authenticity of all cultures was routinely monitored and validated using short tandem repeat (STR) DNA fingerprinting, and tested regularly for mycoplasma. DIPG and adult GBM cultures were cultured as described previously $[14,35,40]$; briefly, all tumor cultures were maintained as neurospheres in Tumor Stem Media (TSM) consisting of DMEM/F12 (Invitrogen), Neurobasal(-A) (Invitrogen), B27(-A) (Invitrogen), human-bFGF (20 ng/ $\mathrm{ml}$ ) (Shenandoah Biotech, Warwick, PA), human-EGF (20 ng/ml) (Shenandoah), human PDGF-AB (20 ng/ml) (Shenandoah) and heparin $(10 \mathrm{ng} / \mathrm{ml})$ (STEMCELL Technologies). Human neural precursor cell cultures were maintained as neurospheres in DMEM/F12, Neurobasal(-A), B27(-A), human-bFGF, human-EGF, heparin, and LIF (Millipore).

\section{Luminex assay}

Neurospheres were dissociated into a single cell suspension, and resuspended at $200 \mathrm{k}$ cells $/ \mathrm{mL}$ in $2.5 \mathrm{~mL}$ TSM without growth factors. After $24 \mathrm{~h}$, supernatants were collected, centrifuged and filtered through a $0.22 \mu \mathrm{m}$ filter to remove cells, aliquoted and immediately stored at $-80^{\circ} \mathrm{C}$. All cultures were collected in triplicate at different cell passages. Supernatants were analyzed on the Luminex 200 platform by the Human Immune Monitoring Center at Stanford University. Human 63-plex kits were purchased from eBiosciences/Affymetrix and used according to the manufacturer's recommendations with modifications. Briefly: Beads were added to a 96-well plate and washed in a Biotek ELx405 washer. Samples were added to the plate containing the mixed antibody-linked beads and incubated at room temperature for $1 \mathrm{~h}$ followed by overnight incubation at $4{ }^{\circ} \mathrm{C}$ with shaking. Cold and Room temperature incubation steps were performed on an orbital shaker at 500-600 rpm. Following the overnight incubation plates were washed in a Biotek ELx405 washer and then biotinylated detection antibody added for $75 \mathrm{~min}$ at room temperature with shaking. Plate was washed as above and streptavidin-PE was added. After incubation for $30 \mathrm{~min}$ at room temperature wash was performed as above and reading buffer was added to the wells. Each sample was measured in duplicate. Plates were read using a Luminex 200 instrument with a lower bound of 50 beads per sample per cytokine. Custom assay Control beads by Radix Biosolutions are added to all wells.

\section{Immunohistochemistry and light microscopy}

Primary tumor samples were processed as previously described [34]. Briefly, primary tumor samples were fixed in $4 \%$ paraformaldehyde-PBS overnight, and then transferred to $30 \%$ sucrose until tissue samples sank (2-3 d). Tissue samples were transferred to cryomolds and embedded in optimal-cutting temperature (OCT) compound (TissueTek). $10 \mu \mathrm{m}$ cryosections were generated on a cryostat (Leica). Endogenous peroxidase activity was neutralized (Bloxall, Vector Laboratories) before samples were permeabilized (0.5\% Triton X-100, TBS) and blocked (5\% horse serum, Vector). Immunohistochemical labeling was performed for CD3 (Biolegend, 300302, 3 h RT). CD3 was developed with a peroxidase secondary (ImmPRESS VR anti-rabbit IgG, Vector) and ImmPACT AMEC Red Peroxidase Substrate (Vector). Tissue samples were counterstained with Hematoxylin QS (Vector, $45 \mathrm{~s}, \mathrm{RT}$ ), developed in a bluing solution $\left(0.1 \% \mathrm{NaCO}_{3} / \mathrm{H}_{2} \mathrm{O}, 1 \mathrm{~min}\right)$ and coverslipped with Fluoro-Gel with Tris buffer (Electron Microscopy Services). Samples were imaged on a Nikon Eclipse. 
Immunofluorescence and confocal microscopy

Primary tumor samples were prepared as described for immunohistochemistry. Frozen cryosections were rehydrated in PBS. Antigen retrieval was performed with L.A.B. Solution (Polysciences, $5 \mathrm{~min}, \mathrm{RT}$ ). Sections were blocked for $1 \mathrm{~h}$ at RT in 3\% NDS (Jackson ImmunoResearch) and stained overnight at $4{ }^{\circ} \mathrm{C}$ with rabbit anti-IBA1 (Wako, 1:1000). Secondary antibodies conjugated with AlexaFluor 488 were used at $\mathrm{RT}$ for $4 \mathrm{~h}$ to detect primary labeling (Jackson ImmunoResearch, 1:500). Sections were mounted in ProLong Gold Antifade Mountant with DAPI (Thermo Fisher). Mounted samples were images using confocal microscopy (Zeiss LSM710), and acquired Z stacks were flattened through maximum intensity projection (Zeiss ZEN).

\section{Results}

We obtained primary DIPG tissue and pediatric normal cortical tissue samples at the time of early post-mortem autopsy (Additional file 1: Table S1). For single cell studies of DIPG, samples were obtained at the time of new diagnosis biopsy as previously described [8]. Adult GBM tissue samples were obtained from early post-mortem autopsy and from surgical biopsies. All tissue collection was performed with informed consent and IRB approval. Immunofluorescence staining of primary DIPG and adult GBM tissue demonstrates a marked increase of Iba1+ stained microglia/macrophages, which exhibit an activated morphology (shorter processes, enlarged cell bodies) when compared to microglia in normal cerebral cortex (Fig. 1a-c). To gain a better understanding of these DIPG-associated microglia/macrophages, we established a
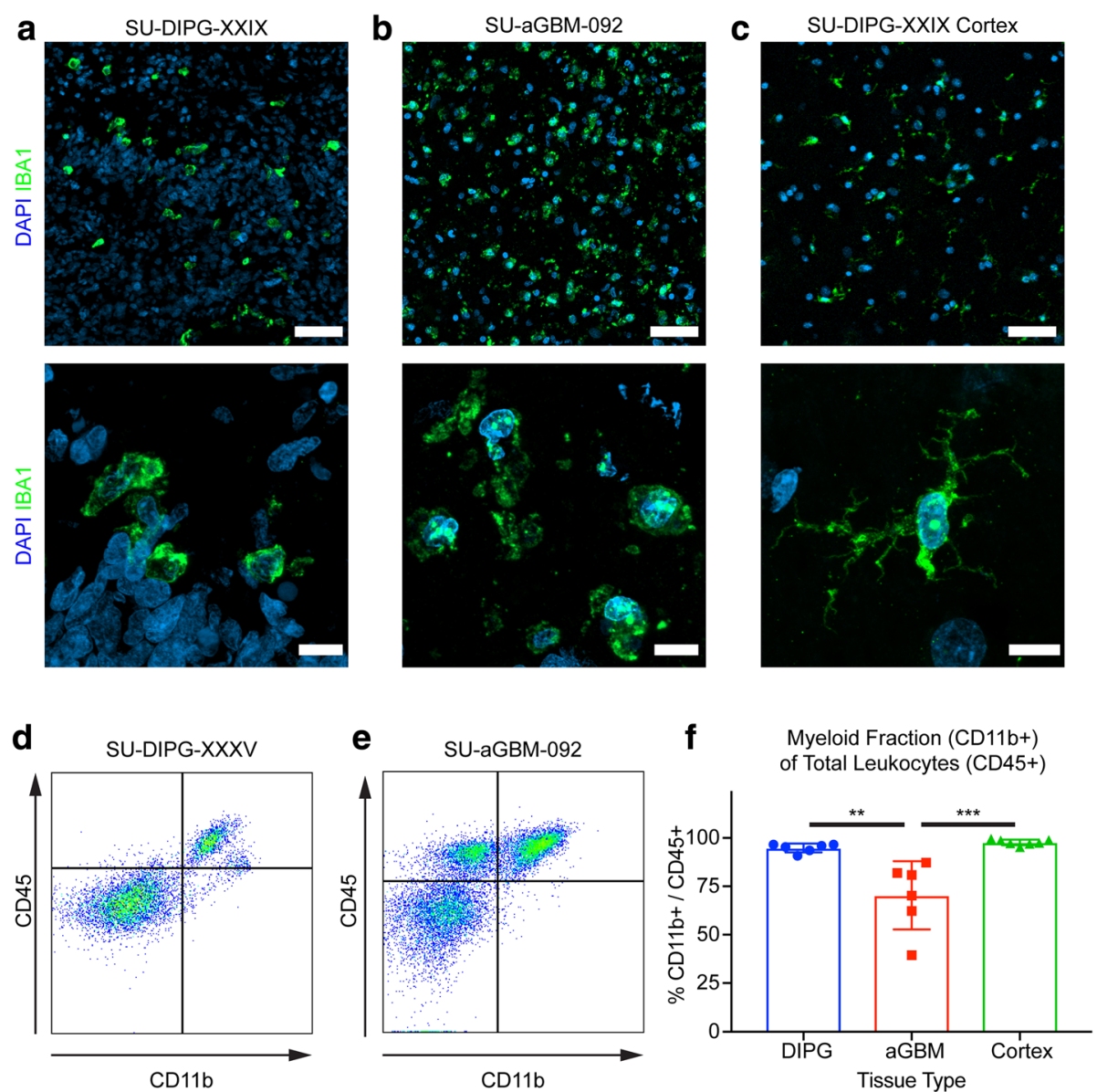

Fig. 1 DIPG and aGBM tumor-associated microglia/macrophages (a-c) Immunofluorescence of primary DIPG tumor tissue (a), primary adult GBM tumor tissue (b), and primary pediatric cerebral cortex (c) for the myeloid cell marker IBA1 (green) and DAPI counterstain (blue). The tumorassociated macrophages in $a-b$ exhibit an amoeboid morphology with shorter processes and enlarged cell bodies, while the normal cortical microglia in c demonstrates the ramified morphology typical of "resting" microglia. Top scale bar $=50 \mu \mathrm{m}$; bottom scale bar $=10 \mu \mathrm{m}$ (d-e) Representative FACS plots showing CD11b expression against CD45 expression of primary DIPG (d) and aGBM (e) tissue. Samples were gated on size, singularity, and viability prior to these plots (f) Quantification of myeloid fraction (CD11 b+) of total leukocytes (CD45+) as calculated by flow cytometry in DIPG, aGBM, and normal cortex primary tissue. ${ }^{* *} p<0.005,{ }^{* * *} p<0.0005$ by t-test with Tukey multiple comparison correction 
fluorescence-activated cell sorting (FACS) protocol for the isolation of $\mathrm{CD} 11 \mathrm{~b}+/ \mathrm{CD} 45+$ cells from early post-mortem autopsy tissue (Fig. 1d-e). Notably, while microglia and peripheral macrophages are sometimes differentiated by CD45 expression levels [1], we did not observe consistently variable CD45-positivity in primary DIPG samples (Additional file 2: Figure S1). We did observe a larger CD45+/CD11b- population in our adult GBM samples, indicating the presence of non-myeloid immune cells in adult GBM. Quantifying the percentage of CD45+ leukocytes that were CD11b + myeloid cells, we found a significant difference between DIPG and adult GBM samples (DIPG: $94.80 \% \pm 0.92 \%$ vs. adult GBM: $70.34 \% \pm 7.20 \%, p$ $<0.005$, Fig. 1f). Nearly all CD45+ cells in normal pediatric cortical tissue were $\mathrm{CD} 11 \mathrm{~b}+$ myeloid cells $(97.71 \% \pm 1.48 \%)$, likely representing normal cortical microglia. As earlier reports exploring the immune compartments of other pediatric gliomas suggest that pediatric high-grade gliomas have limited lymphocytic infiltrate [15, 37], we performed immunohistochemical staining on our tissue samples for CD3 and found minimal CD3 staining in primary DIPG tissue compared to adult GBM, which may account for some of the CD45+/CD11b- population (Fig. 2a-c). Concordantly, flow cytometry revealed a small number of $\mathrm{CD} 3+$ lymphocytes in a representative cohort of DIPG samples compared to adult GBM samples (DIPG: 1.72-2.65\% of total CD45+ leukocytes; adult GBM: 7.0950.2\%; normal cortex: 0.26-1.97\%; Fig. 2d-f). In a separate set of samples, we examined unbiased single-cell RNA sequencing of H3K27M mutant pediatric diffuse midline glioma pre-treatment, diagnostic biopsy samples [8], and identified only 15 lymphocytes out of 2259 total live cells sequenced (0.66\%) (Fig. 2g). These findings together provide further evidence for a minimal lymphocytic infiltrate in DIPG.

To further examine and compare the DIPG- and aGBM-associated macrophage phenotype, we performed gene expression analyses. First, we isolated CD11b+/CD45 + cells from broadly sampled primary DIPG autopsy samples and aGBM tumor autopsy or surgical biospy samples by FACS, verified population purity by flow cytometry, and lysed cells in Trizol for RNA sequencing. After filtering samples for RNA quality, we included six DIPG autopsy, four adult GBM (three surgical biopsy and one autopsy sample), and three pediatric autopsy cortical control samples in our final analyses. These samples all exhibited robust enrichment for microglia/ macrophage-specific genes, validating the FACS-based myeloid cell isolation strategy (Additional file 3: Figure S2). Principal components analysis of the top 500 varying genes amongst these samples demonstrated three distinct populations, with the first principal component primarily separating normal cortex myeloid cells from both adult GBM and DIPG myeloid cell samples, and the second principal component primarily separating DIPG and adult GBM myeloid cell samples (Fig. 3a). Gene ontology (GO) analysis of the top 50 genes enriched in DIPG and adult GBM myeloid cell samples in $\mathrm{PC} 1$ revealed biological process terms including cell adhesion, angiogenesis, and extracellular matrix organization (Table 1). GO analysis of the top 50 genes enriched in adult GBM myeloid cell samples in PC2 identified terms including monocyte chemotaxis, inflammatory response, immune response, neutrophil chemotaxis, cellular response to interleukin-1, and chemokine-mediated signaling pathway, which may suggest that DIPG- and adult GBM-associated macrophages are differentially inflammatory (Table 1).

We next performed differential gene testing between our sample groups using DESeq2 (Fig. 3b-c). After filtering for significance (adjusted $p$-value $<0.1$ ) and minimum gene expression (mean FPKM $>5$ across all samples), there were 330 differentially expressed genes between DIPG and control (176 up, 154 down); 203 between aGBM and control (164 up, 39 down); and 160 between aGBM and DIPG (108 up, 52 down) (Additional file 4: Table S2). Macrophage response phenotypes have historically been separated into M1 "classical activation" and M2 "alternative activation" states, in which M1 macrophages may represent an anti-tumor response whereas M2 macrophages provide pro-tumorigenic functions, although this delineation is widely held to be insufficient to capture the diversity of macrophage phenotypes [31]. Previous reports have suggested that adult GAMs span the spectrum of M1 and M2 phenotypes [10]. We performed pre-ranked gene-set enrichment analysis on significant differentially expressed genes between DIPG-associated macrophages and cortical microglia and similarly found no significant enrichment for either M1 or M2 defined gene sets [32] (Additional file 5: Figure S3A-B), indicating that DIPG-associated macrophages do not fit neatly into an "M1" or "M2" classification.

We also examined the differentially expressed gene lists by GO analysis. GO analysis of genes upregulated in DIPG-associated macrophages compared to cortical microglia include the terms interferon-gamma-mediated signaling pathway, response to hypoxia, antigen processing and presentation (e.g. HLA-A, HLA-DQA1, HLA-DRA), signal transduction, type I interferon signaling pathway, and positive regulation of angiogenesis. GO analysis of genes downregulated in DIPG-associated macrophages compared to cortical microglia include MyD88-dependent toll-like receptor signaling pathway, regulation of cytokine secretion, and toll-like receptor signaling pathway (Additional file 5: Figure S3C-D). This suggests that DIPG-associated macrophages exhibit some degree of activation, consistent with the observed morphological changes (Fig. 1a-c). However, 


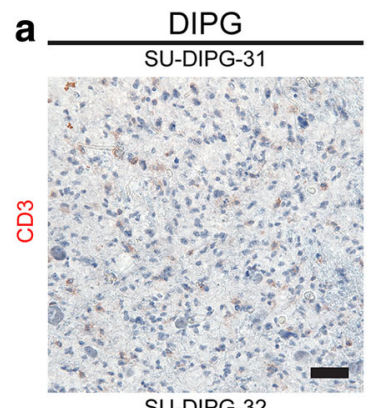

SU-DIPG-32

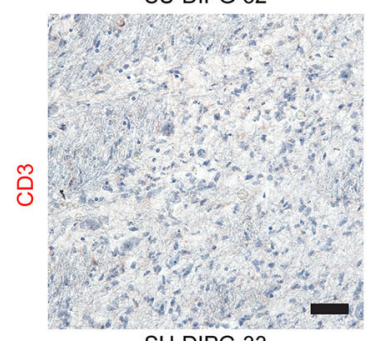
SU-DIPG-33
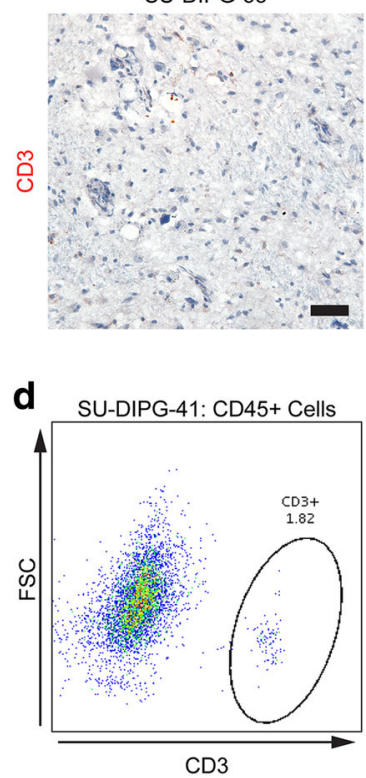

b

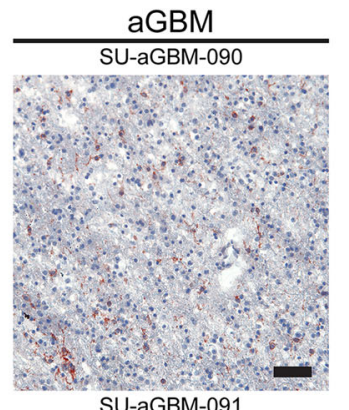

SU-aGBM-091
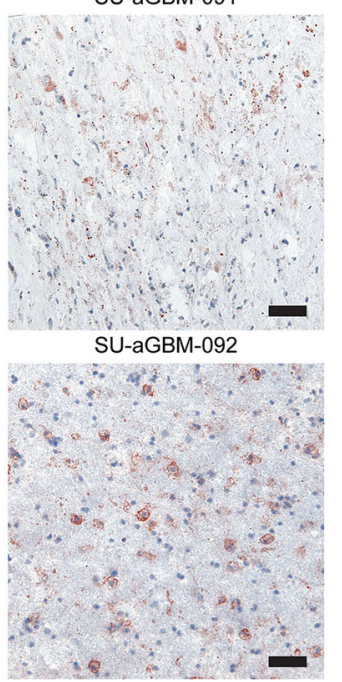

e

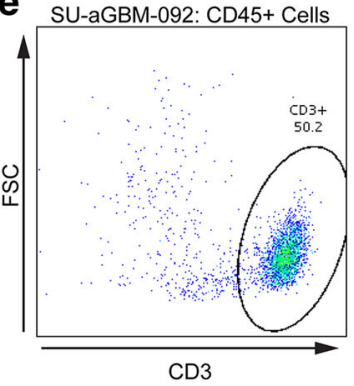

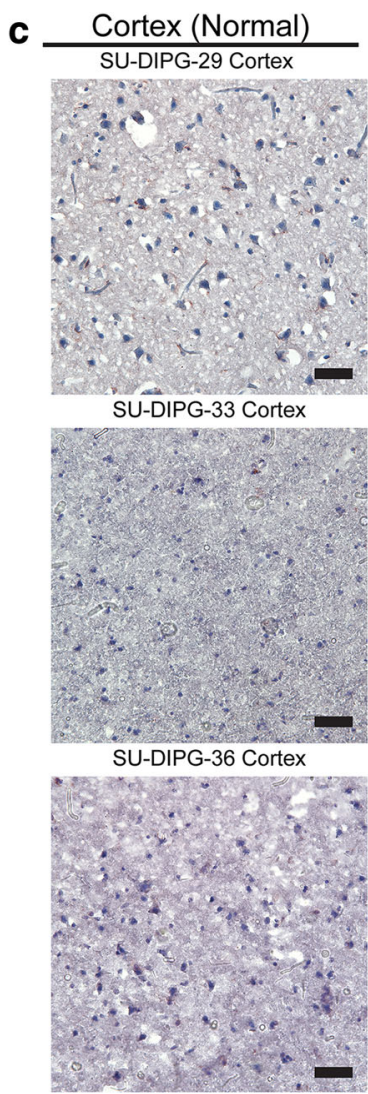

f Lymphocyte Fraction (CD3+)

\begin{tabular}{|c|c|c|} 
of Total Leukocytes (CD $45+)$ \\
\hline DIPG & aGBM & Cortex \\
\hline $2.65 \%$ & $50.20 \%$ & $1.97 \%$ \\
\hline $1.72 \%$ & $7.09 \%$ & $0.26 \%$ \\
\hline & $7.86 \%$ & $0.72 \%$ \\
\hline
\end{tabular}

$\mathbf{g}$

DIPG scRNA Cell Identity

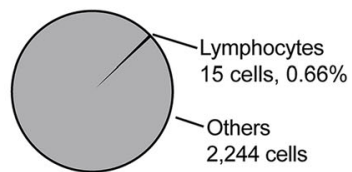

Fig. 2 DIPG and aGBM demonstrate differential CD3+ lymphocyte infiltration (a-c) Immunohistochemical staining for CD3 (red) of primary DIPG (a), adult GBM (b), and pediatric cerebral cortex (c) tissue, counterstained with hematoxylin (blue). Scale bar = 50 $\mu$ m (d-e) FACS plots showing different CD3+ composition of CD45+ cells in DIPG (d) and aGBM (e). Samples were gated for size, singularity, viability, and CD45 positivity prior to these plots (f) Table of lymphocyte (CD3+) fraction of total leukocytes (CD45+) in primary DIPG, aGBM, and pediatric cortical tissue samples as calculated via flow cytometry of primary dissociated early autopsy samples. $\mathbf{g}$ Lymphocyte fraction of all sampled cells from primary single-cell RNA sequencing of DIPG diagnostic biopsy samples [7]

the top genes upregulated in adult GBM-associated macrophages compared to DIPG-associated macrophages include many inflammation-associated genes (e.g. IL6, CCL4, IL1A, IL1B, CCL3, PTGS2) (Fig. 3c). GO analysis of genes upregulated in adult GBM-associated macrophages compared to DIPG-associated macrophages included the terms inflammatory response, positive regulation of smooth muscle cell proliferation, cellular response to interleukin-1, positive regulation of transcription from $R N A$ polymerase II promoter, immune response, cellular response to tumor necrosis factor, and chemokine-mediated signaling pathway (Table 2). Overall, the gene expression profile of adult GBM-associated macrophages and DIPG-associated macrophages are markedly different (Fig. 3d).

Glioma-secreted factors play a role in modulating GAMs and the glioma immune microenvironment [12]. Given that our data suggest DIPG-associated macrophages are less inflammatory than adult GBM-associated macrophages, we 
a

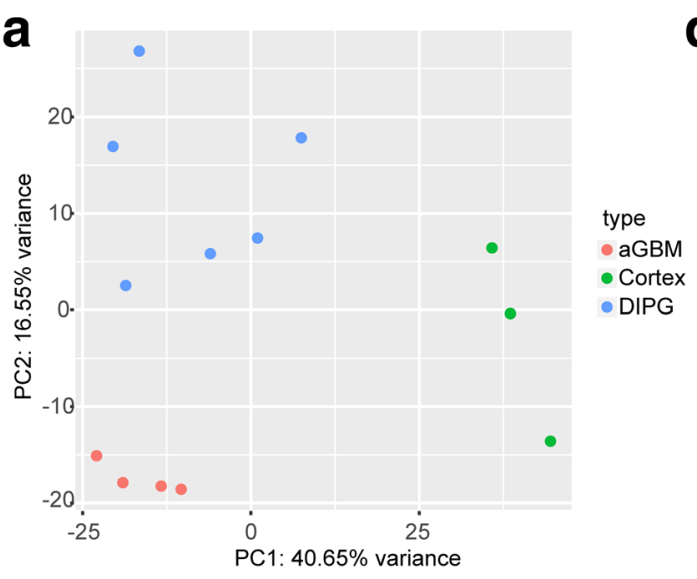

b
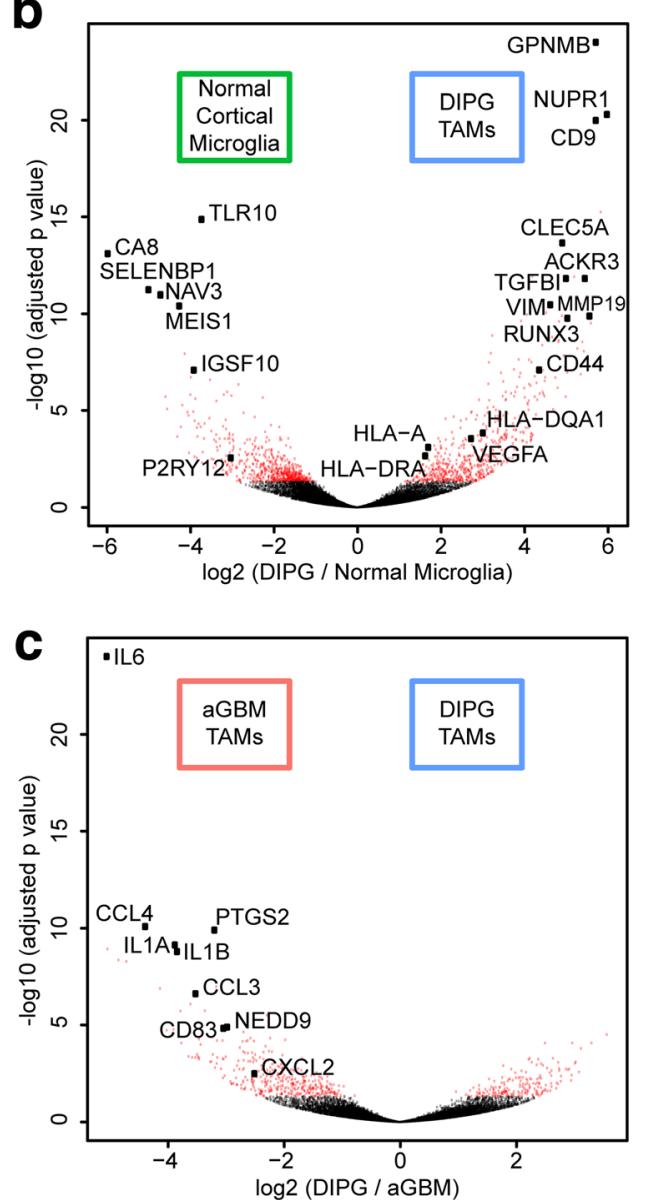

d

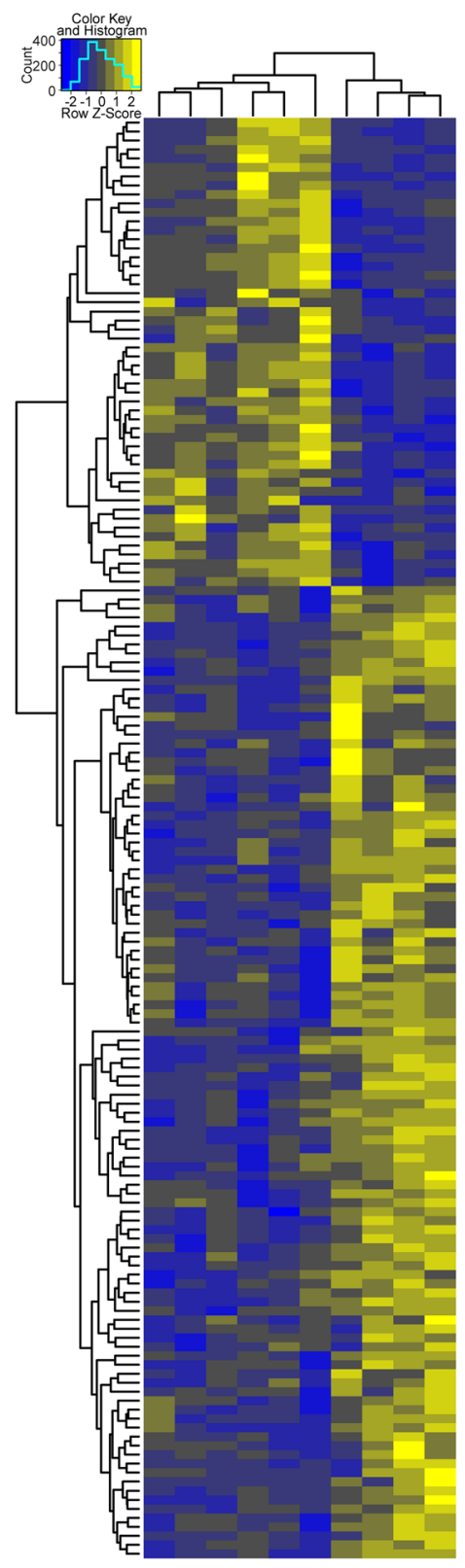

ิํำ

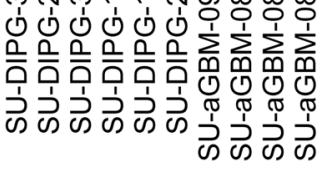

Fig. 3 DIPG- and aGBM-associated macrophages exhibit distinct gene expression profiles (a) Principal components analysis of the top 500 varying genes across all samples demonstrates clusters corresponding to normal cortical microglia (green), DIPG-associated macrophages (blue), and aGBM-associated macrophages (red). b-c Volcano plot of log-fold change against adjusted $p$ value for genes between normal cortical microglia and DIPG-associated macrophages (b), and between DIPG-associated macrophages and aGBM-associated macrophages (c). Red dots represent adjusted $p$ value $<0.05$, and selected significantly differentially expressed genes are identified (d) Heat map of normalized count values for differentially expressed genes between DIPG- and aGBM-associated macrophages. Hierarchical clustering demonstrates a distinct difference between the two groups 
Table 1 Gene-ontology terms associated with the top 50 genes contributing to principal component 1 (top) or principal component 2 (bottom)

\begin{tabular}{|c|c|c|}
\hline \multicolumn{3}{|l|}{ GO Terms: Top genes down in PC1 (up in DIPG/aGBM) } \\
\hline GO Term & $p$-value & Genes \\
\hline Cell adhesion & $3.178 \mathrm{E}-08$ & IBSP, CD9, PLXNC1, CD44, TGFBI, VCAN, ACKR3, THBS1, GPNMB, EMILIN2, CD72, FN1 \\
\hline Angiogenesis & $2.285 \mathrm{E}-06$ & VAV3, EREG, TGFBI, MMP19, CXCL8, ACKR3, ANPEP, FN1 \\
\hline Extracellular matrix organization & $1.488 \mathrm{E}-05$ & IBSP, CD44, DMP1, TGFBI, VCAN, THBS1, FN1 \\
\hline Platelet degranulation & 1.787E-04 & CD9, ACTN1, THBS1, FLNA, FN1 \\
\hline Peptide cross-linking & $3.446 \mathrm{E}-04$ & ANXA1, TGM2, THBS1, FN1 \\
\hline Neutrophil chemotaxis & $7.802 \mathrm{E}-04$ & VAV3, LGALS3, CXCL8, TREM1 \\
\hline Movement of cell or subcellular component & 1.679E-03 & CD9, VIM, CXCL8, FPR3 \\
\hline Platelet activation & 3.834E-03 & CD9, VAV3, ACTN1, FLNA \\
\hline \multicolumn{3}{|l|}{ GO Terms: Top genes down in PC2 (up in aGBM) } \\
\hline GO Term & p-value & Genes \\
\hline Monocyte chemotaxis & $3.14 \mathrm{E}-08$ & IL6, CCL3, CCL2, CCL3L1, CCL4L2, CCL4 \\
\hline Inflammatory response & $9.84 \mathrm{E}-08$ & $P L P 1, I L 6, C C L 3, C C L 2, C C L 3 L 1, C X C L 9, I L 1 B, C C L 4 L 2, C C L 4, I L 1 A$ \\
\hline Immune response & $2.40 \mathrm{E}-07$ & IL6, CCL3, CCL2, ENPP2, FCGR1B, CXCL9, IL1B, CCL4L2, CCL4, IL1A \\
\hline Neutrophil chemotaxis & $3.18 \mathrm{E}-07$ & $C C L 3, C C L 2, C C L 3 L 1, I L 1 B, C C L 4 L 2, C C L 4$ \\
\hline Cellular response to interleukin-1 & 4.60E-07 & IL6, CCL3, CCL2, CCL3L1, CCL4L2, CCL4 \\
\hline Chemokine-mediated signaling pathway & 4.60E-07 & $C C L 3, C C L 2, C C L 3 L 1, C X C L 9, C C L 4 L 2, C C L 4$ \\
\hline Positive regulation of ERK1 and ERK2 cascade & 2.09E-06 & IL6, CCL3, CCL2, CCL3L1, CCL4L2, FGF1, CCL4 \\
\hline Cellular response to tumor necrosis factor & 4.06E-06 & IL6, CCL3, CCL2, CCL3L1, CCL4L2, CCL4 \\
\hline
\end{tabular}

asked whether DIPG-derived factors might differ from adult GBM-derived factors. We collected conditioned medium from 14 patient-derived DIPG cultures, a DIPG culture derived from a frontal lobe metastasis (SU-DIPGXIII-Frontal), two patient-derived pediatric GBM cultures, two human neural precursor cell cultures, and five patient-derived adult GBM cultures and analyzed their cytokine/chemokine secretome using an ELISA array (Luminex 200). Hierarchical clustering analysis showed that the majority of DIPG and pediatric GBM cultures were more like human neural precursor cells and secrete substantially fewer cytokines and chemokines than adult GBM cells (Fig. 4a). Given the low level of DIPG-derived factors, we looked for gene expression of these factors in our previously published patient-derived DIPG cell culture RNA sequencing data $[35,40]$ and found that patient-derived DIPG cell cultures do not express cytokine genes, and only express a limited number of chemokines and growth factors (Fig. 4b, Additional file 6: Figure S4A). Exploring the same genes in primary DIPG tissue bulk RNA sequencing data [14] similarly demonstrated low levels of these cytokines in the overall DIPG tumor

Table 2 Gene-ontology terms upregulated in adult GBM tumor-associated macrophages compared to DIPG tumor-associated macrophages

\begin{tabular}{|c|c|c|}
\hline \\
\hline & \multicolumn{2}{|c|}{$\begin{array}{l}\text { GO Terms upregulated in aGBM TAMs vs. DIPG TAMs } \\
\text { GO Term }\end{array}$} \\
\hline & 4.70E-13 & $\begin{array}{l}\text { NFKBIZ, IL6, CCL3, OLR1, PTGS2, CXCL2, CXCL8, NFKB1, NLRP3, CCL5, CCL4, } \\
\text { CCRL2, FOS, IL1B, NAIP, CLEC7A, NFE2L2, TNFAIP3, IL1A }\end{array}$ \\
\hline IO Term & 5.66E-07 & NAMPT, IL6, EREG, PTGS2, HBEGF, NR4A3, CCL5 \\
\hline \multirow{2}{*}{$\begin{array}{l}\text { Cellular response to interleukin-1 } \\
\text { Positive regulation of transcription from RNA polymerase II } \\
\text { promoter }\end{array}$} & $1.55 \mathrm{E}-06$ & ICAM1, IL6, CCL3, CXCL8, NFKB1, CCL5, CCL4 \\
\hline & $1.57 \mathrm{E}-06$ & $\begin{array}{l}\text { EGR1, NAMPT, IL6, EGR2, CCNH, NR4A2, CCNL1, NFKBIA, NFKB1, FOSB, NR4A3, } \\
\text { NLRP3, FOS, ATF3, RGCC, IL1B, NFE2L2, IL1A, KLF4 }\end{array}$ \\
\hline Immune response & 8.70E-06 & GPR183, IL6, CCL3, CXCL2, IL1RN, CXCL8, IL1B, CCL5, SLED1, CCL4, GBP2, IL1A \\
\hline Cellular response to tumor necrosis factor & $1.98 \mathrm{E}-05$ & ICAM1, IL6, CCL3, CXCL8, NFE2L2, CCL5, CCL4 \\
\hline Chemokine-mediated signaling pathway & 2.96E-05 & CCRL2, CCL3, CXCL2, CXCL8, CCL5, CCL4 \\
\hline Positive regulation of gene expression & $5.38 \mathrm{E}-05$ & IL6, LIMS1, CCL3, RGCC, MDM2, IL1B, NFE2L2, KLF4, IL1A \\
\hline
\end{tabular}



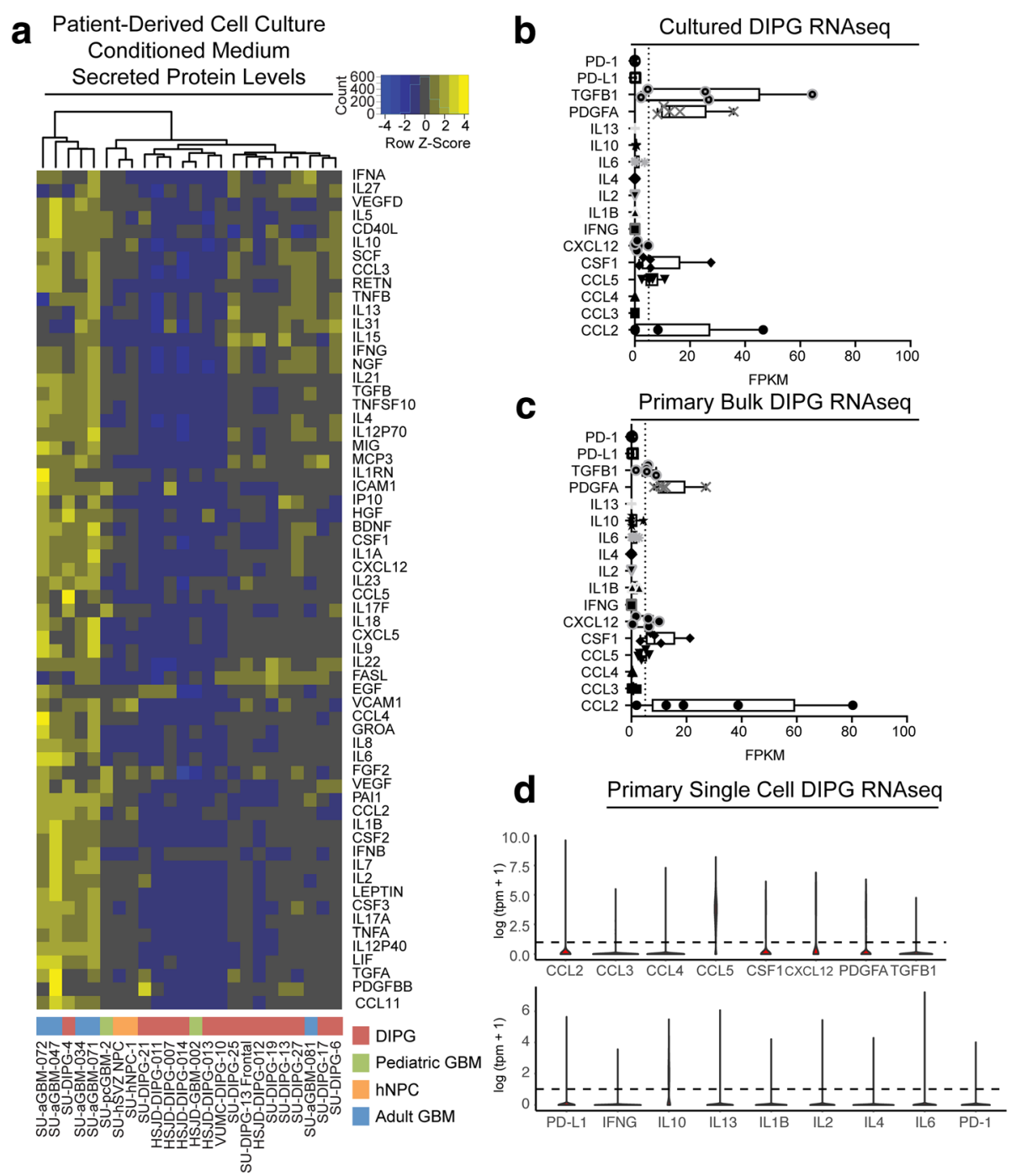

Fig. 4 Patient-derived DIPG and aGBM cell cultures exhibit distinct cytokine secretion profiles (a) Hierarchical clustering of mean fluorescence intensity values of secreted factors in conditioned medium derived from patient-derived DIPG (red), aGBM (blue), pediatric GBM (green), and human neural precursor cell (hNPC) cultures (orange). Each column represents the average of samples tested in triplicate, and each row represents a separate measured cytokine (b-c) Box and whisker plot of FPKMs of cytokines and chemokines from patient-derived DIPG cell cultures (b) and primary bulk DIPG tissue (c) RNA sequencing data [11, 30, 35]. Dashed line represents FPKM =5 (d) Violin plots of gene expression from single-cell RNA sequencing of diagnostic DIPG biopsy samples [7]. Dashed line represents $\log (\mathrm{tpm}+1)=1$

microenvironment (Fig. 4c, Additional file 6: Figure S4B). Finally, we explored these genes in a recent single-cell gene expression dataset that examined primary diagnostic biopsy tissue from DIPG and other H3K27Mmutant diffuse midline gliomas [8], which enables investigating individual DIPG cells in the primary, pretreatment tumor microenvironment (Fig. 4d, Additional file 6: Figure S4C). DIPG tumor cell gene expression data from each of these datasets support the observation that DIPG cells only express a small subset of chemokines and growth factors. Gene transcripts or proteins found in at least three of these four analyses included CCL2, CCL5, CSF1, CXCL12, TGFB1, and PDGFA. Notably, we find that
DIPG cells do not express inflammatory cytokines and chemokines that may recruit other immune cells to the tumor microenvironment. For instance, the lack of IL2 expression by DIPG cells may contribute the lack of T-lymphocyte infiltration observed. Moreover, examination of these gene expression datasets for the immunomodulatory ligands PD-1 and PD-L1 indicated no expression (Fig. 4b-d, Additional file 6: Figure S4).

\section{Discussion}

The data presented here indicate that DIPG-associated macrophages are strikingly less inflammatory compared to adult GBM-associated macrophages, expressing 
markedly lower levels of IL6, IL1A, IL1B, CCL3, CCL4, among other inflammatory factors. Concordantly, we find that DIPG tumor cells in culture, primary autopsy tissue, and pre-treatment diagnostic DIPG biopsy samples, are also non-inflammatory and lack expression of most chemokines and cytokines. In fact, one of the few factors expressed by DIPG is TGFB1, a known immunosuppressive growth factor. We also observe a lack of infiltrating CD3+ lymphocytes in primary DIPG tissue, suggesting that the presence of DIPG does not recruit tumor-infiltrating lymphocytes (TILs). Importantly, we observed this both in early post-mortem DIPG autopsy samples as well as pre-treatment diagnostic DIPG biopsy samples, suggesting that TILs are not a meaningful part of early or late DIPG pathophysiology.

Historically, macrophages have been classified as "M1" classically activated or "M2" alternatively activated phenotypes, although this classification system has been acknowledged to be insufficient to capture the complexity of macrophage responses [31]. Although DIPG tumor cells produce CSF1, a cytokine associated with the M2, pro-tumorigenic phenotype, we found that DIPG-associated macrophages do not fit neatly into an M1 or M2 classification [32]. Similar to reports in adult GBM [10], DIPG-associated macrophages appear to be in a tumor-specific activation phenotype related to the distinct tumor-derived chemokine milieu. In our analysis of DIPG-associated macrophage gene expression, we observed that these cells express increased levels of antigen-presentation genes such as HLA proteins. However, the comparative lack of production of pro-inflammatory chemokines (e.g. CCL3, CCL4) and absence of lymphocytes in primary tissue are consistent with the failure of DIPG-associated macrophages to trigger an effective anti-tumor immune response. Adding to the evidence for lack of an effective innate or adaptive immune response in pediatric high-grade gliomas, a previous study demonstrated the lack of NK cell infiltration into pediatric high-grade gliomas [16], although this study did not specifically investigate DIPG. An "immune cold" state of DIPG is also consistent with the lack of inflammatory cells in pediatric non-brainstem gliomas recently described [30].

The findings presented here are particularly relevant to the development of immunotherapeutic approaches to DIPG. Many current approaches in adult GBM include the use of checkpoint inhibitors [36], the effectiveness of which is linked to pre-existing CD8+ T cell presence [51] and mutational load [42]. Along with our observation that DIPG tumors contain very few infiltrating T-cells, DIPG exhibits a lower mutational burden compared to adult glioblastoma [47]. Thus, immunotherapy approaches in DIPG may be better served by focusing on inducing recruitment or introduction of immune cells to the tumor. One promising strategy involves the use of chimeric antigen receptor $\mathrm{T}(\mathrm{CAR}-\mathrm{T})$ cells, which are designed to recognize tumor-associated antigens. We recently demonstrated striking preclinical efficacy of GD2-targeted CAR-T cell therapy in preclinical models of DIPG [34].

\section{Conclusion}

Adult and pediatric high-grade gliomas are distinct disease entities, and differences between DIPG and adult glioblastoma extend to the immunological phenotype of the tumor microenvironment. In contrast to adult GBM, the immune microenvironment of DIPG is non-inflammatory and does not contain a significant adaptive immune component. These observations provide important considerations for the design of immunotherapeutic approaches for DIPG.

\section{Additional files}

Additional file 1: Table S1. Patient characteristics of early post-mortem DIPG autopsy cases. (XLSX $55 \mathrm{~kb}$ )

Additional file 2: Figure S1. Primary DIPG samples do not consistently demonstrate differential CD45 high/low populations (a-b) Representative FACS plots of primary DIPG tissue samples showing an example of an indistinguishable CD45 high/low sample (a) and a distinguishable CD45 high/low population (b). Samples were gated for size, singularity, and viability prior to these plots. (TIF $585 \mathrm{~kb}$ )

Additional file 3: Figure S2. Isolated microglia/macrophages are enriched for myeloid genes. FPKMs binned across sample type for isolated DIPG (blue), aGBM (red), and pediatric cortical microglia/macrophages (green). There is minimal or absent expression of genes associated with other major cortical cell types (astrocytes, neurons, OPCs, oligodendrocytes, and endothelial cells). (TIF 948 kb)

Additional file 4: Table S2. Significant differentially expressed genes between normal cortical microglia, DIPG-associated macrophages, and aGBM-associated macrophages. (XLSX 75 kb)

Additional file 5: Figure S3. DIPG-associated macrophages are not M1 or M2 (a-b) Pre-ranked gene set enrichment analysis of significantly differentially regulated genes between DIPG-associated macrophages and normal cerebral cortex microglia compared against published gene sets corresponding to M1 (a) or M2 (b) macrophage polarization state [27] (c-d) GO term analysis of upregulated (c) and downregulated (d) genes in DIPG-associated macrophages compared to cortical microglia. (TIF $2553 \mathrm{~kb}$ )

Additional file 6: Figure S4. DIPG cells do not express significant levels of cytokines (a-b) FPKMs of cytokine (left), chemokine (middle) and other factors (right) expressed by patient-derived DIPG cell cultures (a) or in bulk primary DIPG tissue (b) Horizontal line represents FPKM $=5$ (c) Violin plots of single-cell DIPG expression of cytokines, chemokines, and other factors from primary DIPG biopsy tissue. Horizontal line represents $\log (\mathrm{tpm}+1)=1$. (TIF $1442 \mathrm{~kb})$

\section{Acknowledgements}

The authors gratefully acknowledge support from the Cure Starts Now Foundation and DIPG Collaborative, Chan Zuckerberg Initiative and the Silicon Valley Community Foundation, McKenna Claire Foundation, Unravel Pediatric Cancer, Stefan and Julia Roever Research Fund, National Institute of Neurological Disorders and Stroke (NINDS R01NS092597), Child Health Research Institute at Stanford, Anne T. and Robert M. Bass Endowed Faculty Scholarship in Pediatric Cancer and Blood Diseases, the Stanford Medical 
Scientist Training Program, and the Stanford Graduate Program in Neuroscience.

\section{Authors' contributions}

GLL carried out the primary tissue processing, flow cytometry and FACS experiments, RNA extraction and library preparation, gene expression analysis, primary tissue immunostaining, cell culture experiments. SN assisted in RNA gene expression analysis and interpretation. MGF and MLS performed biopsy single-cell isolation and RNA sequencing preparation and analysis. HV assisted with primary tissue immunostaining and interpretation of immunohistochemistry experiments. MM conceived of the study, participated in its design and coordination and supervised all aspects of the work. GLL and MM wrote the manuscript. All authors read and approved the final manuscript.

\section{Ethics approval and consent to participate}

No animals were used in this study. All procedures performed in studies involving human participants were in accordance with the ethical standards of the institutional and/or national research committee and with the 1964 Helsinki declaration and its later amendments or comparable ethical standards.

\section{Consent for publication}

Informed consent was obtained from all individual participants included in the study prior to any tissue collection.

\section{Competing interests}

The authors declare that they have no competing interests.

\section{Publisher's Note}

Springer Nature remains neutral with regard to jurisdictional claims in published maps and institutional affiliations.

\begin{abstract}
Author details
${ }^{1}$ Department of Neurology, Stanford University, Stanford, CA 94305, USA. ${ }^{2}$ Department of Pediatric Oncology, Dana-Farber/Boston Children's Cancer and Blood Disorder Center and Harvard Medical School, Boston, MA, USA. ${ }^{3}$ Department of Pathology, Massachusetts General Hospital, Boston, MA, USA. ${ }^{4}$ Klarman Cell Observatory, Broad Institute of Harvard and Massachussetts Institute of Technology (MIT), Cambridge, MA 02142, USA. ${ }^{5}$ Department of Pathology, Stanford University, Stanford, CA 94305, USA. ${ }^{6}$ Department of Pediatrics, Stanford University, Stanford, CA 94305, USA.
\end{abstract}

\section{Received: 7 June 2018 Accepted: 7 June 2018}

\section{Published online: 28 June 2018}

\section{References}

1. Aguzzi A, Barres BA, Bennett ML (2013) Microglia: scapegoat, saboteur, or something else? Science 339:156-161. https://doi.org/10.1126/science. 1227901

2. Buczkowicz P, Hoeman C, Rakopoulos P, Pajovic S, Letourneau L et al (2014) Genomic analysis of diffuse intrinsic pontine gliomas identifies three molecular subgroups and recurrent activating. Nat Genet 46:451-456. https://doi.org/10.1038/ng.2936

3. Butowski N, Colman H, De Groot JF, Omuro AM, Nayak L et al (2016) Orally administered colony stimulating factor 1 receptor inhibitor PLX3397 in recurrent glioblastoma: an ivy Foundation early phase clinical trials consortium phase II study. Neuro-Oncology 18:557-564. https://doi.org/10. 1093/neuonc/nov245

4. Cage TA, Samagh SP, Mueller S, Nicolaides T, Haas-Kogan D et al (2013) Feasibility, safety, and indications for surgical biopsy of intrinsic brainstem tumors in children. Childs Nerv Syst 29:1313-1319. https://doi.org/10.1007/ s00381-013-2101-0

5. Cannarile MA, Weisser M, Jacob W, Jegg A-M, Ries CH et al (2017) Colonystimulating factor 1 receptor (CSF1R) inhibitors in cancer therapy. J Immunotherapy Cancer 5:53. https://doi.org/10.1186/s40425-017-0257-y

6. Caretti V, Sewing ACP, Lagerweij T, Schellen P, Bugiani M et al (2014) Human pontine glioma cells can induce murine tumors. Acta Neuropathol 127:897-909. https://doi.org/10.1007/s00401-014-1272-4

7. Cooney T, Lane A, Bartels U, Bouffet E, Goldman S et al (2017) Contemporary survival endpoints: an international diffuse intrinsic pontine glioma registry study. Neuro-Oncology 19:1279-1280. https://doi.org/10. 1093/neuonc/nox107

8. Filbin MG, Tirosh I, Hovestadt V, Shaw ML, Escalante LE et al (2018) Developmental and oncogenic programs in H3K27M gliomas dissected by single-cell RNA-seq. Science 360:331-335. https://doi.org/10.1126/science.aao4750

9. Fontebasso AM, Papillon-Cavanagh S, Schwartzentruber J, Nikbakht $H_{\text {, }}$ Gerges N et al (2014) Recurrent somatic mutations in. Nat Genet 46: 462-466. https://doi.org/10.1038/ng.2950

10. Gabrusiewicz K, Rodriguez B, Wei J, Hashimoto Y, Healy LM et al (2016) Glioblastoma-infiltrated innate immune cells resemble M0 macrophage phenotype. JCI Insight. https://doi.org/10.1172/jci.insight.85841DS1

11. Gessi M, Gielen GH, Dreschmann V, Waha A, Pietsch T (2015) High frequency of H3F3A (K27M) mutations characterizes pediatric and adult high-grade gliomas of the spinal cord. Acta Neuropathol 130:435-437. https://doi.org/10.1007/s00401-015-1463-7

12. Gieryng A, Pszczolkowska D, Walentynowicz KA, Rajan WD, Kaminska B (2017) Immune microenvironment of gliomas. Lab Investig 97:498-518. https://doi.org/10.1038/labinvest.2017.19

13. Glass R, Synowitz M (2014) CNS macrophages and peripheral myeloid cells in brain tumours. Acta Neuropathol 128:347-362. https://doi.org/10.1007/ s00401-014-1274-2

14. Grasso CS, Tang Y, Truffaux N, Berlow NE, Liu L et al (2015) Functionally defined therapeutic targets in diffuse intrinsic pontine glioma. Nat Med:110. https://doi.org/10.1038/nm.3855

15. Griesinger AM, Birks DK, Donson AM, Amani V, Hoffman LM et al (2013) Characterization of distinct immunophenotypes across pediatric brain tumor types. J Immunol 191:4880-4888. https://doi.org/10.4049/jimmunol.1301966

16. Haberthur K, Brennan K, Hoglund V, Balcaitis S, Chinn H et al (2016) NKG2D ligand expression in pediatric brain tumors. Cancer Biology \& Therapy 17: 1253-1265. https://doi.org/10.1080/15384047.2016.1250047

17. Hambardzumyan D, Gutmann DH, Kettenmann H (2016) The role of microglia and macrophages in glioma maintenance and progression. Nat Neurosci 19:20-27. https://doi.org/10.1038/nn.4185

18. Huang DW, Sherman BT, Lempicki RA (2009) Bioinformatics enrichment tools: paths toward the comprehensive functional analysis of large gene lists. Nucleic Acids Res 37:1-13. https://doi.org/10.1093/nar/gkn923

19. Huang DW, Sherman BT, Lempicki RA (2009) Systematic and integrative analysis of large gene lists using DAVID bioinformatics resources. Nat Protoc 4:44-57. https://doi.org/10.1038/nprot.2008.211

20. Jansen MHA, Van Vuurden DG, Vandertop WP, Kaspers GJL (2012) Diffuse intrinsic pontine gliomas: a systematic update on clinical trials and biology. Cancer Treat Rev 38:27-35. https://doi.org/10.1016/j.ctrv.2011.06.007

21. Johung TB, Monje M (2016) Diffuse intrinsic pontine glioma: new pathophysiological insights and emerging therapeutic targets. Curr Neuropharmacol 47:156-161.

22. Khuong-Quang D-A, Buczkowicz $P$, Rakopoulos $P$, Liu X-Y, Fontebasso AM et al (2012) K27M mutation in histone H3.3 defines clinically and biologically distinct subgroups of pediatric diffuse intrinsic pontine gliomas. Acta Neuropathol 124:439-447. https://doi.org/10.1007/s00401-012-0998-0

23. Kieran MW (2015) Time to rethink the unthinkable: upfront biopsy of children with newly diagnosed diffuse intrinsic pontine glioma (DIPG). Pediatr Blood Cancer 62:3-4. https://doi.org/10.1002/pbc.25266

24. Kim D, Pertea G, Trapnell C, Pimentel H, Kelley R et al (2013) TopHat2: accurate alignment of transcriptomes in the presence of insertions, deletions and gene fusions. Genome Biol 14:R36. https://doi.org/10.1186/gb-2013-14-4-r36

25. Liao Y, Smyth GK, Shi W (2014) featureCounts: an efficient general purpose program for assigning sequence reads to genomic features. Bioinformatics 30:923-930. https://doi.org/10.1093/bioinformatics/btt656

26. Lin GL, Monje M (2017) A protocol for rapid post-mortem cell culture of diffuse intrinsic pontine glioma (DIPG). JoVE. https://doi.org/10.3791/55360

27. Louis DN, Perry A, Reifenberger G, Deimling A, Figarella-Branger D et al (2016) The 2016 World Health Organization Classi cation of tumors of the central nervous system: a summary. Acta Neuropathol 131:803-820. https:// doi.org/10.1007/s00401-016-1545-1

28. Love MI, Huber W, Anders S (2014) Moderated estimation of fold change and dispersion for RNA-seq data with DESeq2. Genome Biol 15:550. https:// doi.org/10.1186/s13059-014-0550-8

29. Mackay A, Burford A, Carvalho D, Izquierdo E, Fazal-Salom J et al (2017) Integrated molecular meta-analysis of 1,000 pediatric high-grade and diffuse intrinsic pontine glioma. Cancer Cell:1-24. https://doi.org/10.1016/j.ccell. 2017.08.017 
30. Mackay A, Burford A, Molinari V, Jones DTW, Izquierdo E et al (2018) Molecular, pathological, radiological, and immune profiling of nonbrainstem pediatric high-grade glioma from the HERBY phase II randomized trial. Cancer Cell 33:829-842.e5. https://doi.org/10.1016/j.ccell.2018.04.004

31. Martinez FO, Gordon S (2014) The M1 and M2 paradigm of macrophage activation: time for reassessment. F1000 Prime Rep. https://doi.org/10. 12703/P6-13

32. Martinez FO, Gordon S, Locati M, Mantovani A (2006) Transcriptional profiling of the human monocyte-to-macrophage differentiation and polarization: new molecules and patterns of gene expression. J Immunol 177:7303-7311. https://doi.org/10.4049/jimmunol.177.10.7303

33. Mootha VK, Lindgren CM, Eriksson K-F, Subramanian A, Sihag S et al (2003) PGC-1alpha-responsive genes involved in oxidative phosphorylation are coordinately downregulated in human diabetes. Nat Genet 34:267-273. https://doi.org/10.1038/ng1180

34. Mount CW, Majzner RG, Sundaresh S, Arnold EP, Kadapakkam M et al (2018) Potent antitumor efficacy of anti-GD2 CAR T cells in H3-K27M+ diffuse midline gliomas. Nat Med. https://doi.org/10.1038/s41591-018-0006-X

35. Nagaraja S, Vitanza NA, Woo PJ, Taylor KR, Liu F et al (2017) Transcriptional dependencies in diffuse intrinsic pontine glioma. Cancer Cell 31:635-652.e6. https://doi.org/10.1016/j.ccell.2017.03.011

36. Nduom EK, Weller M, Heimberger AB (2015) Immunosuppressive mechanisms in glioblastoma. Neuro-Oncology 17(Suppl 7):vii9-vii14. https:// doi.org/10.1093/neuonc/nov151

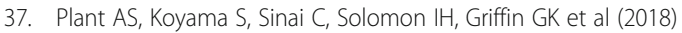
Immunophenotyping of pediatric brain tumors: correlating immune infiltrate with histology, mutational load, and survival and assessing clonal T cell response. J Neuro-Oncol 137:269-278. https://doi.org/10.1007/s11060017-2737-9

38. Puget S, Beccaria K, Blauwblomme T, Roujeau T, James S et al (2015) Biopsy in a series of 130 pediatric diffuse intrinsic pontine gliomas. Childs Nerv Syst 31:1773-1780. https://doi.org/10.1007/s00381-015-2832-1

39. Pyonteck SM, Akkari L, Schuhmacher AJ, Bowman RL, Sevenich L et al (2013) CSF-1R inhibition alters macrophage polarization and blocks glioma progression. Nat Med 19:1264-1272. https://doi.org/10.1038/nm.3337

40. Qin EY, Cooper DD, Abbott KL, Lennon J, Nagaraja S et al (2017) Neural precursor-derived Pleiotrophin mediates subventricular zone invasion by glioma. Cell 170:845-859.e19. https://doi.org/10.1016/j.cell.2017.07.016

41. Quail DF, Bowman RL, Akkari L, Quick ML, Schuhmacher AJ, et al. (2016) The tumor microenvironment underlies acquired resistance to CSF-1R inhibition in gliomas. Science 352:aad 3018. doi: https://doi.org/10.1126/science.aad 3018

42. Rizvi NA, Hellmann MD, Snyder A, Kvistborg P, Makarov V et al (2015) Cancer immunology. Mutational landscape determines sensitivity to PD-1 blockade in non-small cell lung cancer. Science 348:124-128. https://doi. org/10.1126/science.aaa1348

43. Saha D, Martuza RL, Rabkin SD (2017) Macrophage polarization contributes to glioblastoma eradication by combination Immunovirotherapy and immune checkpoint blockade. Cancer Cell 32:253-267.e5. https://doi.org/10. 1016/j.ccell.2017.07.006

44. Schwartzentruber J, Korshunov A, Liu X-Y, Jones DTW, Pfaff E et al (2012) Driver mutations in histone $\mathrm{H} 3.3$ and chromatin remodelling genes in paediatric glioblastoma. Nature:1-8. https://doi.org/10.1038/nature10833

45. Shankar GM, Lelic N, Gill CM, Thorner AR, Van Hummelen P et al (2016) BRAF alteration status and the histone H3F3A gene K27M mutation segregate spinal cord astrocytoma histology. Acta Neuropathol 131:147-150. https://doi.org/10.1007/s00401-015-1492-2

46. Stafford JH, Hirai T, Deng L, Chernikova SB, Urata K et al (2016) Colony stimulating factor 1 receptor inhibition delays recurrence of glioblastoma after radiation by altering myeloid cell recruitment and polarization. NeuroOncology 18:797-806. https://doi.org/10.1093/neuonc/nov272

47. Sturm D, Bender S, Jones DTW, Lichter P, Grill J et al (2014) Paediatric and adult glioblastoma: multiform (epi)genomic culprits emerge. Nat Rev Cancer 14:92-107. https://doi.org/10.1038/nrc3655

48. Sturm D, Witt H, Hovestadt V, Khuong-Quang D-A, Jones DTW et al (2012) Hotspot mutations in H3F3A and IDH1 define distinct epigenetic and biological subgroups of glioblastoma. Cancer Cell 22:425-437. https://doi. org/10.1016/j.ccr.2012.08.024

49. Subramanian A, Tamayo P, Mootha VK, Mukherjee S, Ebert BL et al (2005) Gene set enrichment analysis: a knowledge-based approach for interpreting genome-wide expression profiles. Proc Natl Acad Sci U S A 102:1554515550. https://doi.org/10.1073/pnas.0506580102

50. Taylor KR, Mackay A, Truffaux N, Butterfield YS, Morozova O et al (2014) Recurrent activating ACVR1 mutations in diffuse intrinsic pontine glioma. Nat Genet 46:457-461. https://doi.org/10.1038/ng.2925

51. Tumeh PC, Harview CL, Yearley JH, Shintaku IP, Taylor EJM et al (2014) PD-1 blockade induces responses by inhibiting adaptive immune resistance. Nature 515:568-571. https://doi.org/10.1038/nature13954

52. Vauléon E, Tony A, Hamlat A, Etcheverry A, Chiforeanu DC et al (2012) Immune genes are associated with human glioblastoma pathology and patient survival. BMC Med Genet 5:1-13. https://doi.org/10.1186/1755-87945-41

53. Wu G, Broniscer A, McEachron TA, Lu C, Paugh BS et al (2012) Somatic histone $\mathrm{H} 3$ alterations in pediatric diffuse intrinsic pontine gliomas and non-brainstem glioblastomas. Nat Publ Group 44:251-253. https://doi.org/ 10.1038/ng.1102

54. Wu G, Diaz AK, Paugh BS, Rankin SL, Ju B et al (2014) The genomic landscape of diffuse intrinsic pontine glioma and pediatric non-brainstem high-grade glioma. Nat Genet 46:444-450. https://doi.org/10.1038/ng.2938

\section{Ready to submit your research? Choose BMC and benefit from:}

- fast, convenient online submission

- thorough peer review by experienced researchers in your field

- rapid publication on acceptance

- support for research data, including large and complex data types

- gold Open Access which fosters wider collaboration and increased citations

- maximum visibility for your research: over $100 \mathrm{M}$ website views per year

At BMC, research is always in progress.

Learn more biomedcentral.com/submissions 